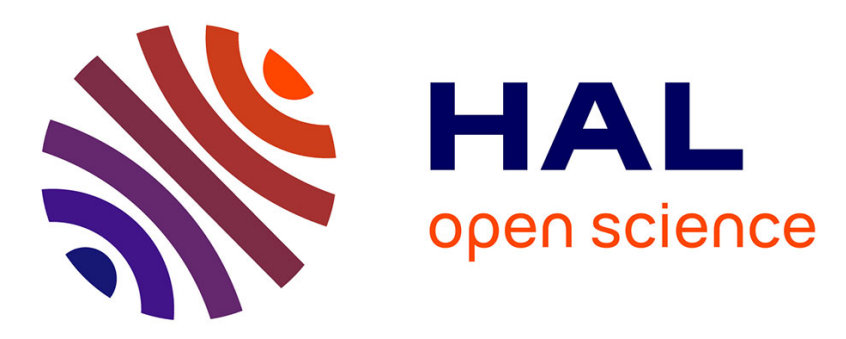

\title{
Tomographic diffractive microscopy of transparent samples
}

\author{
Bertrand Simon, Matthieu Debailleul, Vincent Georges, Vincent Lauer, \\ Olivier Haeberlé
}

\section{- To cite this version:}

Bertrand Simon, Matthieu Debailleul, Vincent Georges, Vincent Lauer, Olivier Haeberlé. Tomographic diffractive microscopy of transparent samples. European Physical Journal: Applied Physics, 2008, 44, pp.29-35. 10.1051/epjap:2008049 . hal-00869566

\section{HAL Id: hal-00869566 https://hal.science/hal-00869566}

Submitted on 2 Dec 2013

HAL is a multi-disciplinary open access archive for the deposit and dissemination of scientific research documents, whether they are published or not. The documents may come from teaching and research institutions in France or abroad, or from public or private research centers.
L'archive ouverte pluridisciplinaire HAL, est destinée au dépôt et à la diffusion de documents scientifiques de niveau recherche, publiés ou non, émanant des établissements d'enseignement et de recherche français ou étrangers, des laboratoires publics ou privés. 


\title{
Tomographic diffractive microscopy of transparent samples
}

\author{
B. Simon ${ }^{1}$, M. Debailleul ${ }^{1}$, V. Georges ${ }^{1}$, V. Lauer ${ }^{2}$, and O. Haeberlé ${ }^{1, a}$ \\ 1 Laboratoire MIPS-LabEl, IUT Mulhouse, 61 rue A. Camus 68093 Mulhouse Cedex, France \\ 2 Lauer Microscopie, 1bis rue des Blés, 68200 Mulhouse, France
}

Received: 13 September 2007 / Accepted: 17 October 2007

Published online: 16 April 2008 - (C) EDP Sciences

\begin{abstract}
We report a tomographic diffractive microscope, which permits imaging non-labelled transparent or semi-transparent samples. Based on a combination of microholography with a tomographic illumination, our set-up creates 3-D images of the index of refraction distribution within the sample. One acquires successively interferograms, rotating the illumination (the specimen being static) and using phase-shifting holography. Within the first Born approximation, each interferogram is interpreted as a subset of the Fourier transform of the specimen index of refraction distribution. The reconstruction is therefore similar to synthetic aperture imaging: one recombines the information in the Fourier space, and a final Fourier transform gives a 3-D image of the specimen. First recalling the theoretical foundations, we then describe our experiment, and show initial results obtained on biological samples.
\end{abstract}

PACS. 42.30.Wb Image reconstruction; tomography - 42.30.Kq Fourier optics - 42.40.Kw Holographic interferometry; other holographic techniques

\section{Introduction}

The optical microscope has become an invaluable tool for biological research thanks to its unique capabilities to image living specimens in three dimensions, and over long periods. Fluorescence techniques often gain preference, because of the specific labelling of cellular structures they allow, thanks to the tremendous variety of available fluorescent dyes. Fluorescence may however be poised off by photobleaching and phototoxicity effects, and labelling may be difficult in itself. As a consequence, techniques permitting the observation of a specimen without the need for specific staining have recently known a regain of interest, as for example, Second-Harmonic or ThirdHarmonic Generation microscopy (SHG, THG), Coherent Anti-Stokes Raman Spectroscopy microscopy (CARS) and even the conventional transmission microscopy and its derivatives. In classical, phase-contrast or Differential Interference Contrast (DIC) microscopy however, the image is the result of a complex interaction of the illuminating light (which is incoherent) with the observed specimen. As a consequence, the recorded gray-levels, while helpful for morphological studies, do not yield quantitative information on the opto-geometrical characteristics of the sample. For example, the specimen optical index of refraction distribution is difficult to reconstruct.

The use of coherent light illumination on the contrary permits to record holograms, which encode both the am-

\footnotetext{
a e-mail: olivier.haeberle@uha.fr
}

plitude and the phase of the light, which is diffracted by the specimen. A model of the diffraction then permits the reconstruction of the specimen index of refraction distribution. For example, within the first order Born approximation domain of validity, the interferogram is interpreted as a subset of Fourier transform of the specimen index of refraction distribution.

Combining this technique with either a rotation of the specimen or an inclination of the illumination wave then represents a diffractive tomographic acquisition, which permits to record a much more complete subset, and therefore allows for 3-D reconstructions of much better quality. Tomographic diffractive microscopy is therefore a true 3D technique, which gives access to an information, which is not, or hardly, available to more conventional microscopies. Furthermore, this technique also allows for a better resolution.

After briefly recalling in the next section the theoretical foundations of this technique, we describe the set-up we built, which is based on a commercial inverted microscope body, and then present results obtained with various specimens.

\section{Theoretical foundations}

The description we give is based on the description of the diffraction phenomenon as given by Wolf [1], within the first Born approximation domain of validity, namely for 
weakly scattering objects. When illuminated by an incoming wave $\Psi_{i}$, of wave vector $\mathbf{k}_{\mathbf{i}}$, such an object only diffracts a small proportion of the incoming light, and $\Psi_{d}$ describes the part of $\Psi_{i}$, which is scattered along all directions.

The diffracted field is decomposed as a sum of plane waves of wavevectors $\mathbf{k}_{\mathbf{d}}$. The elasticity condition (linear optics) implies for each $\mathbf{k}_{\mathbf{d}}$ vector that:

$$
\left\|\mathbf{k}_{\mathbf{d}}\right\|=\left\|\mathbf{k}_{\mathbf{i}}\right\| \text {. }
$$

It results that the set of the $\mathbf{k}_{\mathbf{d}}$ extremity positions depicts a spherical surface, namely the Ewald sphere.

Wolf [1] demonstrated that for a weakly scattering object illuminated by a plane wave, and in the reciprocal space, the wave vectors are linked by:

$$
\mathbf{k}_{\mathbf{d}}=\mathbf{k}_{\mathbf{i}}+\mathbf{k}_{\mathbf{o}}
$$

The radius of the Ewald sphere in the Fourier space is linked to the wavevector $k=2 \pi / \lambda$, where $\lambda$ is the wavelength of observation.

Figure 1a shows the principle of microholography. The wave diffracted by the weakly scattering object is recorded in both amplitude and phase using a holographic recording set-up. However, the set of Fourier components, which can be recorded, is limited by the numerical aperture of the microscope objective $\left(\mathrm{NA}_{o b j}\right)$ used in the detection system (for the sake of clarity, Fig. 1a depicts a 2-D, $\left(k_{y}-\right.$ $k_{z}$ ) representation only (the construction in the $(x-z)$ plane is done the same way). Figure 1e depicts the same process, in the $\left(k_{x}-k_{y}\right)$ plane. In three dimensions, the set of detected waves corresponds to a cap of the Ewald sphere, limited by the detection numerical aperture).

For a given incident wavevector $k_{i}$, and an observation direction $k_{o}$, the corresponding object wavevector is therefore $k_{d}-k_{i}$ ( $-k_{i}$ is indicated by dotted-dashed arrows in Fig. 1). The set of detection therefore describes a dotted arc of circle in Figure 1a and a dotted disk, which is centred about the origin in Figure 1e.

Note that in transmission microholography, the subset, which is recorded is very limited, as only one cap of sphere is recorded. The consequences of this limited acquisition are to be described in Section 4 .

The idea of coherent diffractive tomographic microscopy is to increase the set of components, which can be recorded, in order to perform a more accurate reconstruction of the object from a more complete subset of object components than in microholography.

A possible solution consists in keeping the illumination and detection static, while rotating the specimen $[2,3]$. This set-up presents the advantage that the optical interferometer has no moving parts, which is favourable in terms of vibrations or alignments, but keeping a precise rotation at the microscopic scale may be difficult. Furthermore, this set-up necessitates a special preparation of the observed sample, which has to be embedded within a rotating microcapillary. This procedure is often not favoured by biologists, who usually prefer to prepare and observe their biological samples between a coverglass and a glass slide. Rotating the microcapillary also obliges
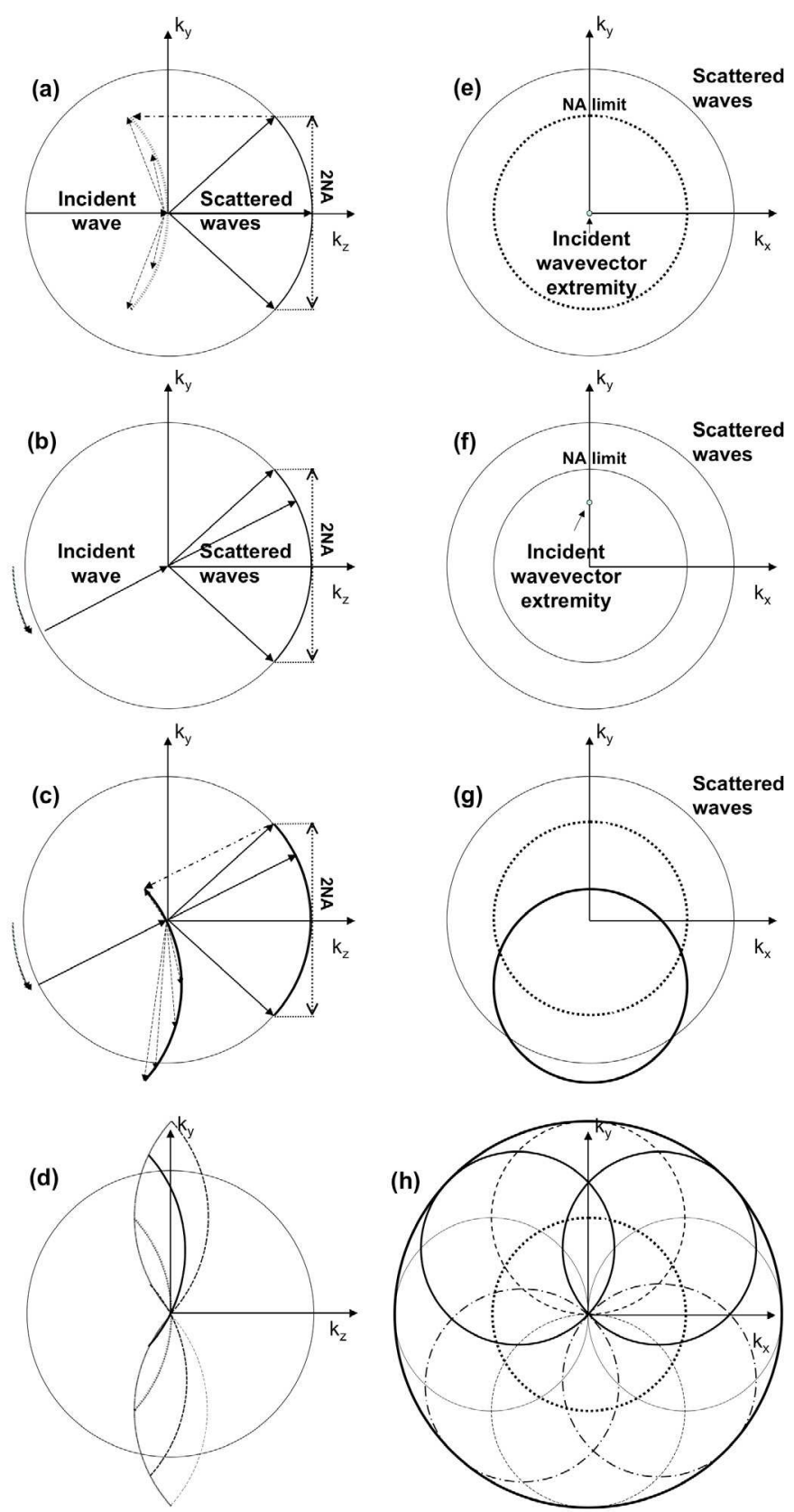

Fig. 1. Principle of diffractive tomographic microscopy: construction of the detected frequency support.

to use longer working distance objectives, which have a lower numerical aperture. As a consequence, the final resolution is expected to be lower (at least the lateral resolution, the main advantage of this set-up being to provide an isotropic resolution, so that the longitudinal resolution provided by tomography with sample rotation is better than the longitudinal resolution of tomography with illumination rotation. See Sect. 4 for an illustration of the anisotropic resolution of tomography with illumination rotation). 
It seems therefore preferable to keep the specimen static. For technical reasons, it is easier to rotate the illumination set-up than to rotate the detection set-up, which would need to rotate both the reference beam and the collected diffracted beam, which have to interfere. In our approach, the detection system and the specimen are therefore fixed, and tomographic acquisition is performed by changing the angle of incidence of the illumination wave. This is achieved using a high NA condenser, as in a classical incoherent transmission microscope.

In Figure $1 \mathrm{~b}, 1 \mathrm{f}$ the direction of $k_{i}$ is changed, therefore the diffracted wavevectors, while the recording directions are the same as in Figures 1a, 1e. However, after proper shifting with respect to the incident wavevector, another set of the object wavevectors are detected as shown in Figures 1c, 1g.

Figures 1d,1h show the different sets of components that are obtained in the $(x, z)$ and $(x, y)$ planes for normal incidence and for 8 incidences corresponding to the two maximum polar angles allowed by the numerical aperture of the condenser and four azimuth angles varying from $0^{\circ}$ to $360^{\circ}$ every $45^{\circ}$ (for the sake of simplicity, we consider that $\mathrm{NA}_{\text {cond }}$ is equal to $\left.\mathrm{NA}_{o b j}\right)$. For example, the upper dashed disk in Figure $1 \mathrm{~h}$ corresponds to an illumination at $90^{\circ}$ azimuth angle. It shows up as the upper dashed arc of circle in Figure 1d. When a large number of incidences is used, the support of the detected components in the $(x, y)$ plane becomes the disk limited by the bold circle in Figure $1 \mathrm{~h}$. It is worth noting that the extension of the domain obtained by varying the incidence angle (the bold circle in Fig. 1h) is twice that given by a digital holographic microscope where sole normal incidence is used (dotted circle in Fig. 1h).

In the $(y, z)$ plane, the Fourier domain scanned by the diffractive tomographic microscope when a large number of incident angles is used takes the form of the well-known butterfly shaped support of the Optical Transfer Function (OTF) for a transmission microscope, which limits are shown in Figure 1d.

Finally, equation (2), which physically corresponds to the momentum conservation equation, can be directly rewritten in terms of Fourier spatial frequencies, which is more easily interpretable in terms of image processing, as:

$$
\nu_{\mathbf{d}}=\nu_{\mathbf{i}}+\nu_{\mathbf{o}}
$$

This equation indicates that within the first order Born approximation, it is possible from the recorded diffracted wave to reconstruct a two-dimensional, spherical subset of the three-dimensional frequency representation of the weakly scattering object: diffraction is simply interpreted as a Fourier transformation [1,4].

In the Fourier plane, one then records Fourier components of the object index of refraction distribution. The interest of using a tilted illuminating waves is that it permits both to record higher frequencies from the Fourier transform of the specimen, as well as to fill the set of detected waves at lower frequencies. It therefore appears interesting to use as high illumination angles as possible, therefore the use of a high NA condenser.
When the extended set of frequencies is recorded by tomography, a final 3-D numerical Fourier transform then permits to directly reconstruct a $3-\mathrm{D}$ image of the specimen. Note that this image is complex, the measured complex values corresponding to the complex index of refraction, depicting a purely dephasing effect, but also possible absorption by the specimen, within the limits of the Born approximation, namely that the objects are weakly absorbing only.

Also interesting is to note the parallel, which exists between tomographic microscopy and synthetic aperture radar imaging: both techniques are based on recording the amplitude and the phase of the diffracted wave, a proper recombination of data acquired under different acquisition conditions, and a final numerical reconstruction, therefore the name of synthetic aperture holographic microscopy sometimes used to describe coherent diffractive tomographic microscopy [5].

Within this paper, we restrict our approach to the first order Born approximation, which greatly simplifies the numerical processing of the data, as only 3-D Fourier transforms are involved. Other reconstruction methods do exist [6], which permit to extend the domain of application of tomography for example to objects with a high contrast, for which multiple scattering must be taken into account [7]. Evanescent waves may be used to illuminate the diffracting specimen, which permits to increase the set of object frequencies, which are recorded, and therefore allows for a better reconstruction of the observed specimen, with a resolution beyond the Abbe limit [8]. A priori information about the specimen may also lead the reconstruction algorithm towards better results [9]. For weakly scattering biological objects however, the reconstruction method we use has proven to give good results [10].

\section{Experimental set-up}

Few results from such tomographic microscopes using coherent wave have been reported in the literature.

Charrière et al. [2] have published results obtained using the technique of sample rotation, combined with an inverse Radon transform to perform the specimen rotation. More recently, Gorsky and Osten [11] have built a set-up in which both the specimen and the detection arm can rotate to increase the set of detected frequencies.

Kawata et al. [12] had built a tomographic microscope set-up by modifying a transmission microscope, but their approach is limited to purely dephasing (i.e. nonabsorbing) specimens. For such samples, theory indeed explains that the Fourier transform of the specimen is symmetric with respect to the origin. This permits a great simplification of the system, as interferograms are taken for extremal illuminating waves only (corresponding to the highest angles allowed by the condenser). The data acquisition is then performed by rotating the impinging wave using a Pechan prism, and the full butterfly-shaped support is reconstructed by symmetry.

This approach being limited to purely dephasing objects, the technique developed by Lauer [10] seems 


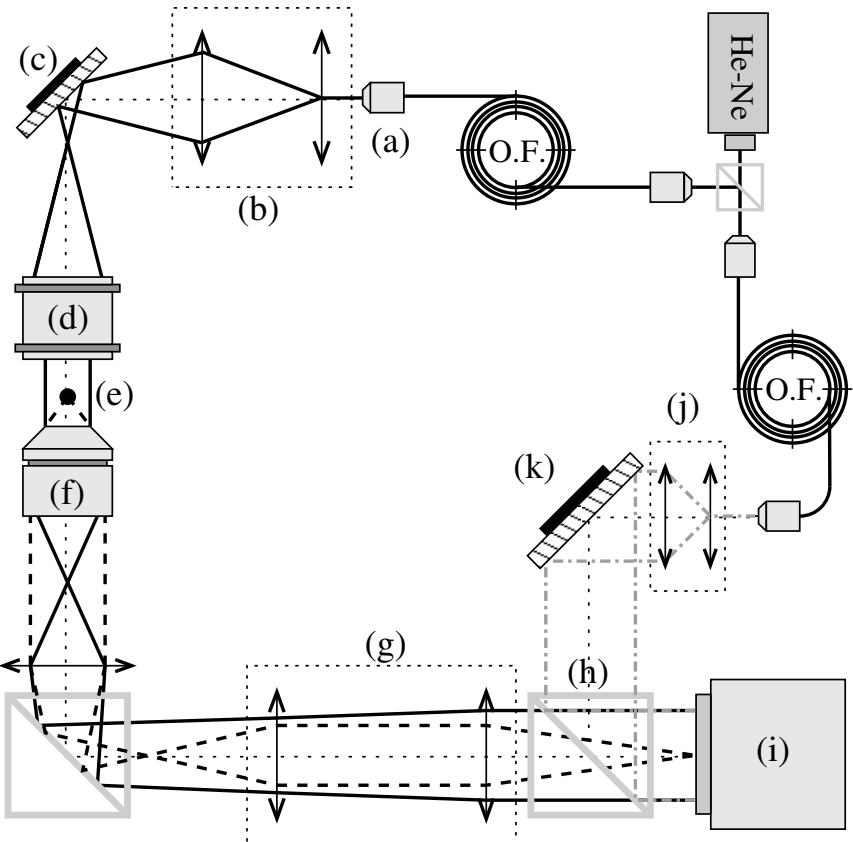

Fig. 2. Sketch of the tomographic diffractive microscope. (a) optical fiber exit. (b) lens set. (c) tilting mirror. (d) condenser. (e) specimen location. (f) objective. (g) lens set. (h) beam recombinator. (i) CCD camera. (j) lens set. (k) shifting mirror.

preferable in most cases. We have adapted this technique to a modern, infinity-corrected microscope, also keeping the possibility to perform fluorescence acquisitions. Figure 2 describes our set-up, and is to be compared with Figure 1 of reference [10]. Our microscope is built using an Olympus IX71 inverted microscope body. A HeNe laser $(\lambda=633 \mathrm{~nm})$ generates the coherent beam, which is split $(50 / 50 \%)$ into an illumination wave and a reference wave that are injected into optical fibers. Optical fibers are used in order to simplify the transport of the illumination beam towards the condenser. We have designed a new condenser holder and illumination system, replacing the standard thermal lamp by an optical bench, which role is to spatially filter and refocus the illumination beam onto the back focal plane of the condenser. As a consequence, the illumination wave leaves the condenser as a plane wave.

The direction of this illumination wave is controlled using a tilting mirror, the tilt being greatly amplified by the condenser, so as to cover the full range of illumination angles for a high numerical, oil immersion condenser $\left(\mathrm{NA}_{\text {cond }}=1.4\right)$. Passing through the specimen, the illumination wave is partly diffracted. The oil immersion objective $\left(100 x, \mathrm{NA}_{o b j}=1.4\right)$ collects the diffracted wave (dotted line), and focuses it at infinity. The Telan lens refocuses it to form an image onto the CCD detector. The non-scattered part of the incoming wave is also captured by the objective (solid line). The telescope after the Telan lens is adjusted so as to have this non-scattered part being refocused as a plane wave when impinging onto the CCD sensor, as well as to properly rescale the image in order to satisfy the sampling theorem. A recombination cube is used to mix the scattered- as well as the non-scattered

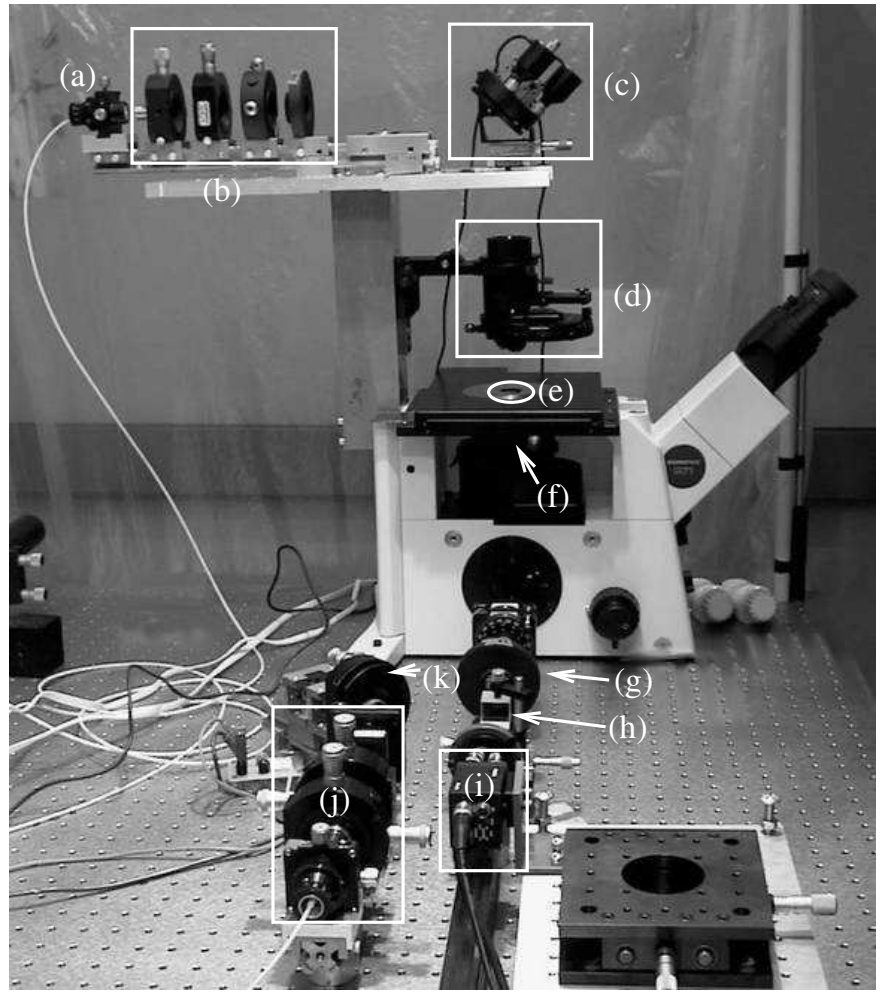

Fig. 3. Practical implementation of tomographic microscopy on an IX71 Olympus inverted microscope body. The large footprint of the set-up is to be reduced in a future version, combined with confocal fluorescence acquisition (see conclusion).

beams with the reference beam, shaped as a plane wave, in order to record holograms. The phase of this hologram is reconstructed with a four-step phase shifting procedure, using a controlled piezo-electric mirror [13].

A numerical Fourier transform of the image then gives the data to be tiled onto the cap of the Ewald sphere corresponding to the illumination (see Fig. 1). This point constitutes a major difference with the original approach of Lauer [10], who performed a direct acquisition into the Fourier space, as a lens indeed performs a Fourier transform of the field when using coherent light. This approach presented the following drawback: the peak corresponding to the zero order is of much higher intensity than the diffracted components. As a consequence, Lauer had to use controlled beam attenuators to perform a double acquisition in the Fourier space: one with low intensity, in order to record the zero-order peak and the low frequencies without saturating the camera, and a second at higher intensity, saturating the low frequency region, but allowing to record the higher frequency components. Both holograms were then numerically restitched.

At this point it is interesting to compare with the Köhler illumination in a transmission microscope. In classical incoherent microscopy, the non-scattered part of the illumination waves appears as a parasitic diffuse luminous background, which renders the observation of the scattered part difficult in the case of weakly diffusing specimens. In our case, this non-scattered beam on the contrary 


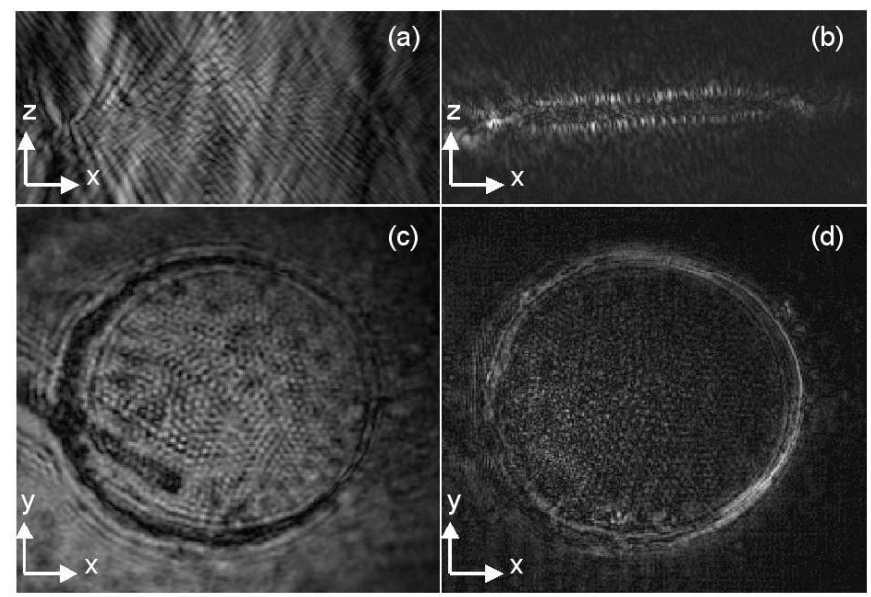

Fig. 4. Reconstructed images of a diatom cell wall (Coscinodiscus $s p$ ) with only one incidence angle in $x-y$ plane (a) and in $x-z$ plane (b), and with 374 incidence angles in $x-y$ plane (c) and in $x-z$ plane (d). Axis arrows represents $5 \mu \mathrm{m}$

constitutes the key-point of the synthetic aperture process. Indeed, the sequential retrieval, for numerous illuminations, of both the amplitude and phase of the diffracted field is a major hurdle for a correct numerical reconstruction of the specimen: the phase between the illumination and reference beams may change because of mechanical vibrations, thermal drifts, or simply because the optical path of the illumination changes with the illumination angles.

Furthermore, the amplitude may also vary. We compensate for these fluctuations by simply normalizing our computed holograms, in both amplitude and phase, with respect to the null frequency, which in fact corresponds to the illumination beam frequency.

Figure 3 shows the Olympus IX71 inverted microscope body, equipped with our tomographic system. Note the large footprint of the tomographic system, which is to be much reduced in an improved version to be developed now that the proof of feasibility has been established, and in order to be able to combine tomographic acquisition with confocal fluorescence onto the same specimen (see Part 4).

\section{Results}

We now briefly present some results obtained with our tomographic set-up with biological samples. Figure 4 compares holographic microscopy for only one illumination incidence along the optical axis and optical diffraction tomography for 372 incidences of illumination. The sample is a diatom cell inner structure (Coscinodiscus sp), which is composed of silica, and therefore mostly transparent. Such specimens are therefore very difficult to observe in standard transmission microscopy (oblique illumination or phase contrast microscopy for example do permit to observe them, but being based on incoherent illumination, the contrast they provide is linked with the optical characteristics of the specimen in a complex way. In particular, these techniques do not, for example, allow for a measurement of the specimen index of refraction). In Figure 4, the contrast is provided simply by depicting the squared modulus of the reconstructed index of refraction.

In Figure 4a characteristic longitudinal diffraction fringes from punctual objects are visible: there is no discrimination along the $z$-axis. This is indeed a major drawback of holographic microscopy: the resolution along the optical axis is very weak. In Figure $4 \mathrm{~b}$ these fringes disappear and the horizontal walls of the diatom cell are now visible. Note also that the other elongated diatom, which appears superimposed on the round diatom in Figure 4c is not visible in Figure 4d. In fact, it belongs to another plane of the 3-D image, which is discriminated in Figure $4 \mathrm{~d}$, whereas it is not in Figure 4c. This is easily explained by comparing the longitudinal extension of the detected frequency support in microholography (see Fig. 1a), which indeed has no thickness, with that of tomographic microscopy (see Fig. 1d). Note also the better lateral resolution visible in Figure 4d, linked to the lateral extension of the detected frequency support in tomography, which is ideally twice that of holography (compare Figs. 1e and 1h). This demonstrates the better 3-D imaging capabilities of diffraction tomographic microscopy, compared to classical holographic microscopy.

Figure 5 illustrates these 3-D imaging capabilities by displaying an 18 -steps, $0^{\circ}$ to $180^{\circ}$ rotation of the specimen. Here, a maximum intensity projection is used, so as to display the entire specimen on each view, and not slices through the specimen as in Figure 4. The lenticular structure of this peculiar diatome is now perfectly visible. Note the elongated structure, visible on the first image because of the maximum intensity projection, which is visibly sticked to the top of the Coscinodiscus sp lenticular diatom, as one can see on the $90^{\circ}$ lateral view. Note also that the lateral resolution is better than the longitudinal resolution. The smallest structures indeed appear as punctual on the "en face" views, while side views do exhibit elongated structures. Our microscope being a transmission-only set-up, a so-called "missing cone" appears in the Optical Transfer Function along the optical axis, and the resolution is lower along that axis. This feature is common to all transmission microscope. In order to obtain an isotropic resolution, a combined reflexiontransmission set-up [10] should be used, very similar to the $4 \mathrm{Pi}[14]$ and $\mathrm{I}^{5} \mathrm{M}$ [15] concepts used in fluorescence microscopy.

Our microscope does however permit the reconstruction of the optical refraction index distribution within the specimen. While currently not yet calibrated, this feature may however be helpful for biologists, as illustrated by Figure 6 . The considered specimen is now snowdrop pollen (Galanthus nivalis). Again, maximum intensity projection images are considered, depicting a $0^{\circ}-90^{\circ}$ rotation of the specimen. The upper-raw displays an autofluorescence image, acquired with an Olympus FV300 confocal system $(100 x, \mathrm{NA}=1.4$ oil immersion objective, $\lambda_{\text {exc }}=543 \mathrm{~nm}$, detection above $\left.560 \mathrm{~nm}\right)$. Note that the auto-fluorescence comes out of the pollen envelope only. 


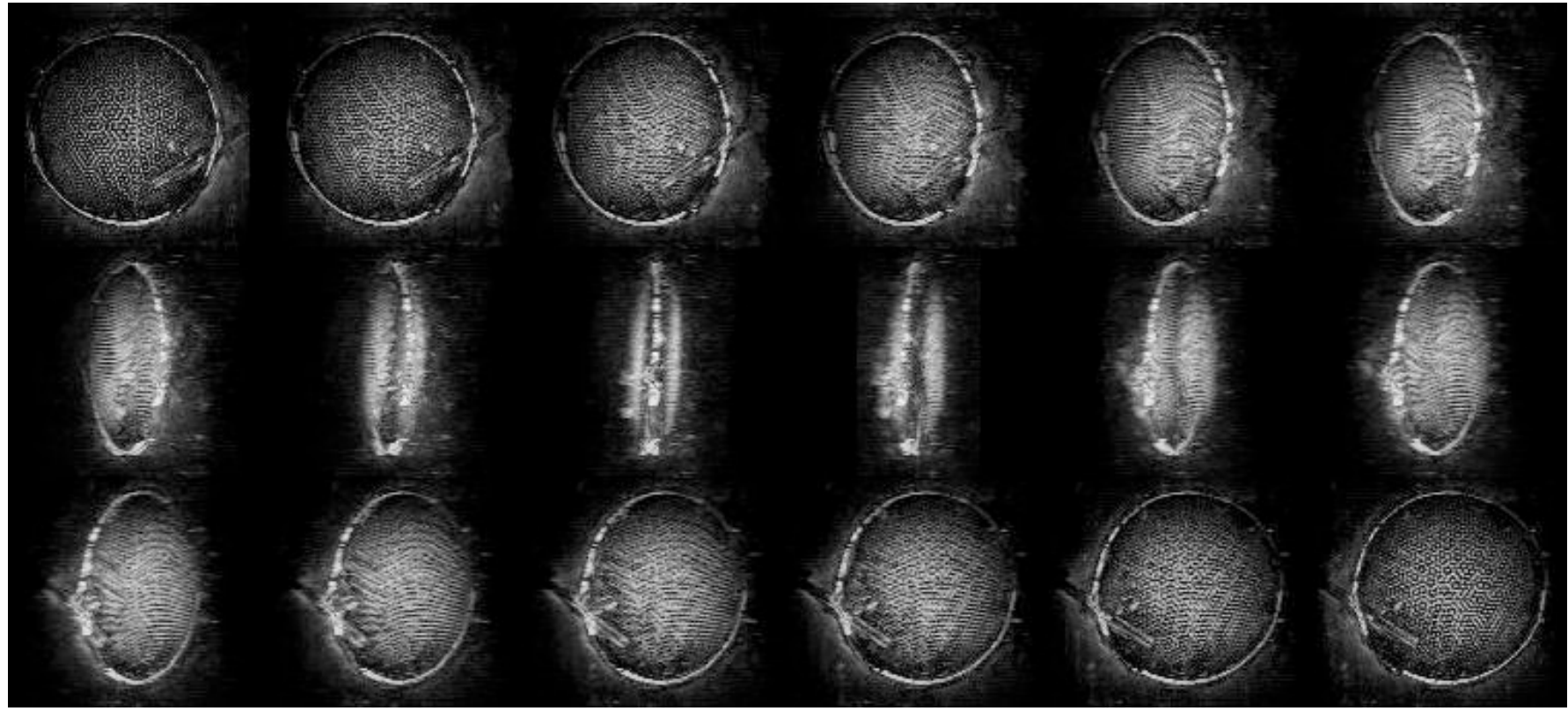

Fig. 5. Three-dimensional view of a Coscinodiscus sp diatome. The pictures depict an 18 -steps, $0^{\circ}-180^{\circ}$ rotation of the specimen. Note the anisotropic resolution, characteristic of transmission microscopes.

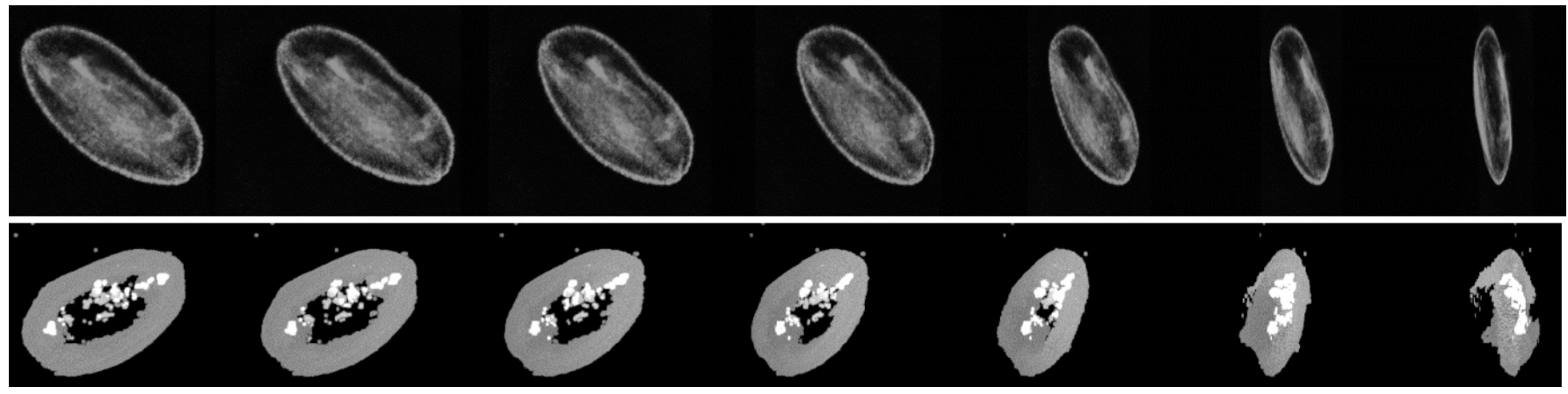

Fig. 6. Upper row: autofluorescence image of a snow drop (Galanthus nivalis) pollen grain. Lower row: tomographic image of a snow drop pollen grain. Both image line display a $90^{\circ}$ rotation of the sample. Note the inner structures, visible on the tomographic image, while not on the autofluorescence image.

The lower image on the contrary shows a tomographic acquisition. It is not the same pollen grain as on the upperrow, the reason being that our tomographic system is not yet equipped for simultaneous confocal acquisition, so that the preparation had to be observed with two different systems. The observed features were however visible on several different pollen grains. The registered squared modulus image has been segmented. The threshold is the mean value of the immersion medium of the specimen, fixed in Eukitt, with $n=1.510$ (Kindler, Freiburg, Germany). The white values depicts measured index of refraction of higher values, the grey level depicts a lower measured index of refraction. Note that the envelope of the pollen is visible, as in the previous image, but now, inner structures, invisible on the fluorescence image, do appear. These structures may correspond to concentrations of high index of refraction sugars usually contained by pollens, and mandatory for the plant germination $[16,17]$. So, even if not calibrated, tomographic microscopy in this case provides access to an information, which is not accessible to autofluorescence images.

\section{Conclusion and perspectives}

We have built a tomographic diffractive microscope, which uses coherent light for holographic imaging, combined with an angular scanning of the illumination in order to allow for the collection of information normally not available to microholography. The key point consists in a correct recombination of the information in the Fourier space, which we perform by using the non-scattered components as a common reference to all acquired holograms.

Our set-up performs very favourably, compared to holographic microscopy, in terms of lateral but mostly 
longitudinal resolution, so that $3-\mathrm{D}$ representation of transparent and semi-transparent specimens is possible.

We have also shown that the measurement of the index of refraction distribution within the specimen may provide a complementary, helpful information for biologists, in association with fluorescence imaging.

From an information processing point of view, the classical transmission microscope may be considered as a parallel information processing device, the specimen being simultaneously illuminated with all incidence angles by the condenser, while the diffractive tomographic microscope with coherent light is a sequential system, where the information is acquired step-by-step, and numerically reconstructed. This point up-to-now constitutes the major drawback of our set-up, which for the moment necessitates several minutes to acquire the many holograms mandatory for a correct 3-D reconstruction of the specimen. As a consequence, live-specimen imaging is not yet possible. We plan to improve our set-up by using fast scanning mirrors, electro-optics phase retardator, and a ultra-fast camera, so as to be able to acquire a $3-\mathrm{D}$ image in less than 1 $\mathrm{mn}$, in order to be compatible with confocal fluorescence 3 -D imaging. Indeed, we want to equip our IX71 microscope with a confocal head, so as to be able to acquire fluorescence and tomographic images of the same specimen, under the same objective.

An important point will be the calibration of the index of refraction distribution, which for the moment is qualitative only (above or below the value of the specimen fixation medium). We plan to use calibrated beads (latex, polymers, glasses) in order to test our system, not only for shape reconstructions, but also for index of refraction measurements.
The authors gratefully acknowledge Dominique Voisin for the diatom samples, as well as Jean-Jacques Delaunay and Stanislas Vertu for enlightening discussions about diffractive tomography and pollens.

\section{References}

1. E. Wolf, Opt. Commun. 1, 153 (1969)

2. F. Charrière et al., Opt. Lett. 31, 178 (2006)

3. M. Fauver et al., Opt. Exp. 13, 4210 (2005)

4. M. Born, E. Wolf, Principles of Optics (Cambridge University Press, Cambridge, 1999)

5. S.A. Alexandrov et al., Phys. Rev. Lett. 97, 168102 (2006)

6. P. Guo, A.J. Devaney, J. Opt. Soc. Am. A 22, 2338 (2005)

7. K. Belkebir, P.C. Chaumet, A. Sentenac, J. Opt. Soc. Am. A 23, 586 (2006)

8. P.C. Chaumet, K. Belkebir, A. Sentenac, Opt. Lett. 29, $2740(2004)$

9. S. Kawata, O. Nakamura, S. Minami, J. Opt. Soc. Am. A 4, 292 (1987)

10. V. Lauer, J. Microsc. 205, 165 (2002)

11. W. Gorski, W. Osten, Opt. Lett. 32, 1977 (2007)

12. T. Noda, S. Kawata, S. Minami, Appl. Opt. 31, 670 (1992)

13. W. Jueptner, U. Schnars, Digital Holography Digital Hologram Recording, Numerical Reconstruction, and Related Techniques (Springer, Berlin, 2005)

14. S.W. Hell, E.H.K. Stelzer, Opt. Commun. 93, 277 (1992)

15. M.G.L. Gustafsson, D.A. Agard, J.W. Sedat, J. Microsc. 195, 10 (1999)

16. M.L. Laucks et al., J. Aerosol Sci. 31, 307 (2000)

17. C.S. Pappas et al., Appl. Spectr. 57, 23 (2003)

To access this journal online: www.edpsciences.org 